\title{
The electromyographic and cephalometric analyses after maxillary expansion*
}

\author{
Patrícia Maria Monteiro ${ }^{1}$, Simone Cecilio Hallak Regalo ${ }^{2}$, Janete Cinira Bregagnolo ${ }^{1}$, \\ Maria Bernadete Sasso Stuani ${ }^{1 \#}$ \\ ${ }^{1}$ Department of Pediatric Clinics, Preventive and Community Dentistry, Division of Orthodontics, Dental School of Ribeirão Preto, \\ University of São Paulo, Ribeirão Preto, Brazil \\ ${ }^{2}$ Department of Morphology, Stomatology and Physiology, Dental School of Ribeirão Preto, University of São Paulo, Ribeirão Preto, \\ Brazil \\ Email: " bernadete@,forp.usp.br
}

Received 9 August 2012; revised 8 September 2012; accepted 17 September 2012

\begin{abstract}
The purpose of this study was to evaluate the electromyographic (EMG) activity of the masseter and temporalis muscles and the skeletal and dental behavior of children having slow maxillary expansion with a quadhelix appliance. The study sample consisted of 30 children (mean age $=7$ years 4 months; 17 girls/13 boys) diagnosed with a unilateral posterior crossbite. Lateral cephalometric tracing and EMG analyses were carried out before orthodontic treatment $\left(T_{1}\right)$ and 1 month after removal of the appliance $\left(T_{2}\right)$. The EMG activity of the masseter and temporalis muscles was analyzed in two clinical situations: at muscular rest and during habitual chewing. Differences in the measurements were evaluated by using paired $t$ tests. Maxillary expansion with the quadhelix appliance did not promote significant anteroposterior and vertical skeletal alterations. Among the cephalometric variables related to dental pattern, only 1-ANS-PNS values presented a significant increase at $\mathbf{T}_{2}$. At muscular rest, EMG analysis indicated a significant increase in the activity of the temporalis muscle. During habitual chewing, EMG activity decreased significantly for both evaluated muscles after removal of the quadhelix appliance. These alterations suggest an adaptation and reprogramming of the musculature to the new occlusal condition.
\end{abstract}

Keywords: Electromyography; Masticatory Muscles; Malocclusion; Orthodontics Interceptive

\section{INTRODUCTION}

The stomatognathic system is a physiological and func-

\footnotetext{
"We gratefully acknowledge the Brazilian agencie CAPES for scholarship financial support.

${ }^{*}$ Corresponding author.
}

tional entity composed of organs and tissues whose biology and physiopathology are interdependent. The anatomic alterations in their organization, which normally affect the dental arches and supporting bones [1], induce the occurrence of compensatory adaptations that impair the functioning of the stomatognathic system as a whole [2]. The harmonic relationship between the maxillary and mandibular dental arches, which is not observed in patients with a posterior crossbite, determines occlusal stability, which, in turn, is related to the normal performance of the musculature [3].

Chewing is the most important function of the stomatognathic system and is the initial phase of the digestive process. If a malocclusion is present, muscular adaptations may occur during the phase of mandibular closure. In fact, patients with a unilateral posterior crossbite submitted to electromyographic analysis of the masseter and temporalis muscles present an altered and asymmetric muscular contraction pattern [4] as well as disturbances of activity $[5,6]$.

Posterior crossbite is a frequent finding in dental clinical practice. The prevalence of this malocclusion in the primary, mixed and permanent dentitions ranges from 8 to 16 percent $[7,8]$. As a posterior crossbite does not improve with the eruption of the successor teeth, the relationship of posterior crossbite in the primary dentition will be similar to that in the permanent dentition $[9,10]$. In addition to not resolving spontaneously, a posterior crossbite compromises aesthetics, occlusal stability, normal oral functions and the overall activity of the masticatory muscles $[5,6]$. Therefore, early treatment is necessary for establishment of the craniofacial balance and consequent myofunctional correction, favoring the child's normal facial growth and development [8].

Correction of a posterior crossbite by slow maxillary expansion is indicated for the primary dentition and precocious mixed dentition because the force used does not 
cause discomfort to the child and high success rates are achieved [11]. This orthodontic therapy allows for a better physiological response and lower recurrence potential when compared with rapid maxillary expansion. The quadhelix appliance is a type of fixed expander often used in pediatric patients [10-13] for correction of unilateral or bilateral posterior crossbites when tooth movement in the buccolingual direction is required [14].

Although EMG analysis of the masticatory muscles provides important data on the functional impact of morphological discrepancies and allows for functional evaluation of treatments for occlusal problems, there are no studies evaluating the behavior of the muscles in children with a posterior crossbite treated by slow maxillary expansion. The cephalometric changes have been extensively investigated, but studies associating the dental changes with myofunctional alterations are limited. Therefore, the purpose of this study was to evaluate the muscular alterations promoted by the treatment of a posterior crossbite with quadhelix appliance in order to assess the masticatory muscle function in the stability of malocclusion correction, as well as to establish any relationship between muscular activity and cephalometric alterations.

\section{SUBJECTS AND METHOD}

Ethical approval for this investigation was granted by the Human Ethical Committee at Ribeirão Preto School of Dentistry, University of São Paulo, Brazil. Thirth patients (17 girls and 13 boys) with mean age of 7 years 4 months, presenting a unilateral posterior crossbite (functional posterior crossbite) and with indication for maxillary expansion as the first stage of the orthodontic treatment were enrolled in the study. Diagnostic of malocclusion was made at maximal habitual intercuspal (MHI) and centric relation (CR). As inclusion criteria, the children should have no previous orthodontic treatment, good general and oral health, with the permanent maxillary and mandibular first molars erupted. Mean time for correction of malocclusion was 3 months and 17 days.

The quadhelix appliance was constructed according to the specifications described by the literature [12]. The initial activation was equivalent to half the buccolingual width of the anchoring molars, which corresponded to an approximate distance of $4.0 \mathrm{~mm}$ at each side. Whenever required, the subsequent activations were performed extraorally at 30-day intervals until crossbite overcorrection was noticed.

Lateral teleradiographs were obtained before the start of treatment $\left(\mathrm{T}_{1}\right)$ and 1 month after removal of the quadhelix appliance $\left(\mathrm{T}_{2}\right)$. For analysis of the anteroposterior behavior of the apical bases, the following cephalometric measurements were used: SNA, SNB, ANB, Ar-A and Ar-Gn. For analysis of the vertical behavior of the apical bases the following cephalometric measurements were used: SN to GoGn, SN to ANS-PNS, ANS-PNS to GoGn, SN to Occlusal Plane (SN.Ocl), SN to Gn, BaN to PtGn, N-ANS, ANS-Me, N-Me, S-Ar, Ar-Go and S-Go. For analysis of the dental behavior of the apical bases, the following cephalometric measurements were used: 1 to NA, $\underline{1-N A}, \underline{1}$-ANS-PNS and $\underline{6}$-ANS-PNS (Table 1).

Table 1. Pre $\left(T_{1}\right)$ and post-treatment $\left(T_{2}\right)$ means and standard deviations (SD) of the cephalometric measurements.

\begin{tabular}{|c|c|c|c|c|c|}
\hline & \multicolumn{2}{|c|}{$\mathrm{T}_{1}$} & \multicolumn{2}{|c|}{$\mathrm{T}_{2}$} & \multirow[b]{2}{*}{$\mathrm{p}$ value } \\
\hline Measurement & Mean & SD & Mean & SD & \\
\hline \multicolumn{6}{|l|}{ Anteroposterior } \\
\hline SNA $\left({ }^{\circ}\right)$ & 81.33 & 3.25 & 81.25 & 3.16 & 0.92 \\
\hline $\operatorname{SNB}\left({ }^{\circ}\right)$ & 77.00 & 2.73 & 77.33 & 3.25 & 0.58 \\
\hline $\operatorname{ANB}\left({ }^{\circ}\right)$ & 4.33 & 2.22 & 3.90 & 1.67 & 0.48 \\
\hline $\operatorname{Ar}-\mathrm{A}(\mathrm{mm})$ & 80.42 & 3.75 & 80.08 & 4.25 & 0.48 \\
\hline $\operatorname{Ar}-\mathrm{Gn}(\mathrm{mm})$ & 96.08 & 4.18 & 96.83 & 5.28 & 0.31 \\
\hline \multicolumn{6}{|l|}{ Vertical } \\
\hline SN to $\mathrm{Gn}\left({ }^{\circ}\right)$ & 68.08 & 2.27 & 68.58 & 2.27 & 0.33 \\
\hline $\mathrm{SN}$ to ANS-PNS $\left({ }^{\circ}\right)$ & 6.75 & 2.73 & 7.33 & 2.22 & 0.50 \\
\hline SN.Ocl $\left({ }^{\circ}\right)$ & 18.75 & 4.11 & 18.75 & 3.57 & 1.00 \\
\hline $\mathrm{SN}$ to $\mathrm{GoGn}\left({ }^{\circ}\right)$ & 34.08 & 3.72 & 34.50 & 4.30 & 0.55 \\
\hline ANS-PNS to GoGn $\left({ }^{\circ}\right)$ & 27.17 & 4.44 & 28.08 & 3.57 & 0.46 \\
\hline $\mathrm{BaN}$ to $\mathrm{PtGn}\left({ }^{\circ}\right)$ & 87.08 & 3.26 & 86.83 & 3.29 & 0.60 \\
\hline $\mathrm{N}-\mathrm{Me}(\mathrm{mm})$ & 107.80 & 6.02 & 106.60 & 4.90 & 0.36 \\
\hline N-ANS (mm) & 45.75 & 3.10 & 46.17 & 2.44 & 0.40 \\
\hline ANS-Me (mm) & 62.17 & 2.85 & 62.00 & 3.38 & 0.74 \\
\hline S-Go (mm) & 67.00 & 3.70 & 66.17 & 3.85 & 0.15 \\
\hline $\mathrm{S}-\mathrm{Ar}(\mathrm{mm})$ & 31.27 & 2.28 & 30.45 & 2.25 & 0.05 \\
\hline Ar-Go (mm) & 39.42 & 2.96 & 39.75 & 2.63 & 0.65 \\
\hline \multicolumn{6}{|l|}{ Dental } \\
\hline$\underline{1}$ to $\mathrm{NA}\left({ }^{\circ}\right)$ & 20.92 & 4.75 & 21.75 & 5.02 & 0.68 \\
\hline$\underline{1}$-NA $(\mathrm{mm})$ & 3.00 & 2.00 & 3.41 & 1.62 & 0.63 \\
\hline 1-ANS-PNS (mm) & 24.92 & 3.82 & 27.08 & 2.10 & $0.01^{*}$ \\
\hline$\underline{6}$-ANS-PNS (mm) & 21.08 & 1.44 & 20.92 & 2.31 & 0.80 \\
\hline \multicolumn{6}{|l|}{ Profile } \\
\hline $\operatorname{ANL}\left({ }^{\circ}\right)$ & 116.8 & 12.53 & 113.3 & 6.42 & 0.28 \\
\hline NA to $\operatorname{Pog}\left({ }^{\circ}\right)$ & 8.66 & 5.82 & 8.08 & 3.91 & 0.68 \\
\hline S-Ls (mm) & 2.58 & 2.15 & 2.33 & 2.10 & 0.58 \\
\hline S-Li (mm) & 2.75 & 2.34 & 2.58 & 1.50 & 0.76 \\
\hline
\end{tabular}

Statistically significant difference if $\mathrm{p}<0.05$ (Student's t-test). 


\subsection{Electromyographic Analysis}

This analysis was performed using a MyoSystem-BR1 (Prosecon, Uberlândia, Brazil) electromyographer. Five channels were used with simultaneous acquisition, common grounding for all channels, bypass filters of $10 \mathrm{~Hz}$ at $5 \mathrm{KHz}$; entrance of $10 \mathrm{G} \Omega$ in a differential mode, 12 bits of dynamic resolution, band of amplitude $-10 \mathrm{~V}$ to $110 \mathrm{~V}$; and sample frequency by channel of $2 \mathrm{KHz}$. Myoeletric signals were captured by various active electrodes with 2 contacts of $10.0 \times 1.0 \mathrm{~mm}$ with a distance of $10.0 \mathrm{~mm}$ apart, impedance upwards of $10 \mathrm{G} \Omega$, and a common rejection value of $130 \mathrm{~dB}$ to $60 \mathrm{~Hz}$, crafted in silver and fixed in a resin capsule of $40 \times 20 \times 5 \mathrm{~mm}$. In order to reduce electromagnetic interferences and other acquisition noises, a $3-\mathrm{cm}^{2}$ stainless steel ground electrode was fixed on the skin over the sternum region.

Surface differential active electrodes were placed on the skin, previously cleaned with alcohol, bilaterally on both masseter muscles and on the anterior portion of the temporalis. The position of the electrodes was determined by palpation, and maximum voluntary contraction was performed to guarantee that the muscles were accurately located.

The EMG signals were captured during muscular rest and habitual chewing for 10 seconds. In order to capture the signals at rest, the patients were asked to remain relaxed without occluding the teeth. For recording habitual chewing, they were instructed to chew a Trident ${ }^{\mathbb{B}}$ chewing gum tablet.

\subsection{Statistical Analysis}

Both cephalometric and electromyographic data were tabulated and analyzed statistically using the Statistical Package for Social Sciences (SPSS Inc.; Chicago, Illinois, USA) version 12.0 for Windows. A student's t-test for repeat measurements was used to compare the means of each variable at $T_{1}$ and $T_{2}$. Significance was set at 5 percent. The errors of the method were calculated using Dahlberg's [15] formula with double measurements of these variables performed on all patients.

EMG signals were visualized and processed by using the program Myosystem-Br1 (version 2.29, Prosecon). After digitalization, the signals were analogically amplified with a 1000 times gain, filtered (bypass filter 0.01 $1.5 \mathrm{kHz}$ ), and sampled with an A/D 12 bites converter plate with a $2 \mathrm{KHz}$ acquisition rate. RMS (root mean square) values at rest and habitual chewing were normalized with the RMS value of clenching at maximum habitual intercuspation: i.e. these values were expressed as a percentage of the maximum voluntary clench. The use of normalized EMG potentials was made so that EMG data could be compared and differences between recordings of the same subject and different subjects could be reduced, thus making interpretation of the data reproducible.

\section{RESULTS}

\subsection{Cephalometric Analysis}

Means and standard deviations of the cephalometric measurements obtained at $\mathrm{T}_{1}$ and $\mathrm{T}_{2}$ are presented in Table 1 .

The evaluation of the anteroposterior and vertical behavior of the apical bases at the end of the treatment did not show statistically significant alterations $(p>0.05)$ in any of cephalometric measurements. Regarding dental behavior, there was an increase $(p<0.05)$ in the means of 1 -ANS-PNS measurements at $T_{2}$.

\subsection{Electromyographic Analysis}

At the end of the orthodontic treatment, there was a significant increase $(p<0.05)$ in EMG activity of the temporalis muscles at rest (Figure 1). The EMG activity of all muscles evaluated during habitual chewing decreased significantly $(\mathrm{p}<0.05)$ at $\mathrm{T}_{2}$ (Figure 2$)$.

\section{Rest Condition}

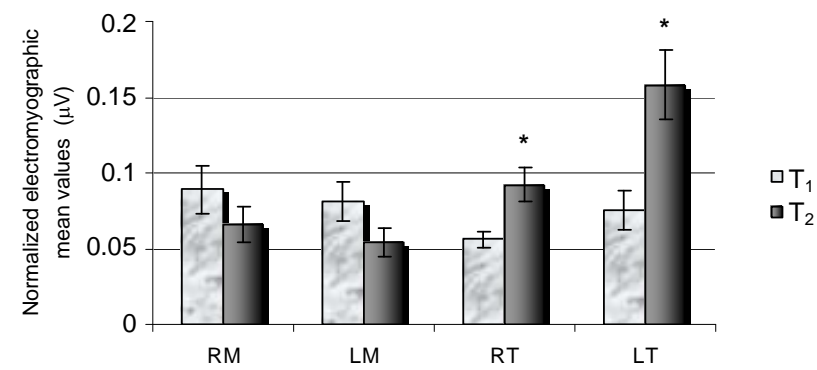

Figure 1. Initial $\left(\mathrm{T}_{1}\right)$ and final $\left(\mathrm{T}_{2}\right)$ normalized electromyographic mean values at muscular rest condition $\left({ }^{*} \mathrm{p}<0.05\right)$. RM: Right Masseter; LM: Left Masseter; RT: Right Temporalis; LT: Left Temporalis.

Rest Condition

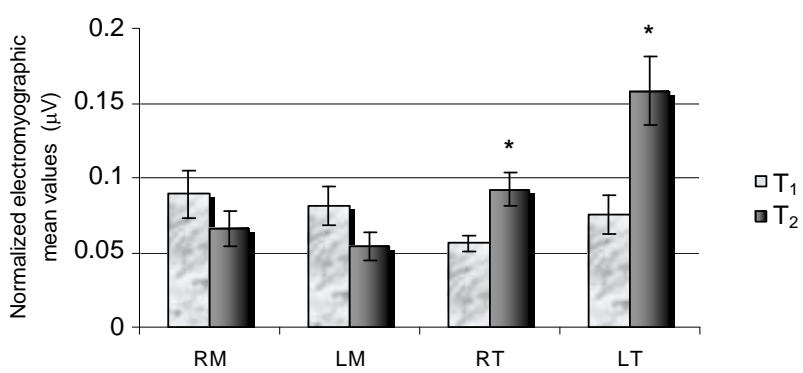

Figure 2. Initial $\left(\mathrm{T}_{1}\right)$ and final $\left(\mathrm{T}_{2}\right)$ normalized electromyographic mean values during habitual chewing $\left({ }^{*} \mathrm{p}<0.05\right)$. RM: Right Masseter; LM: Left Masseter; RT: Right Temporalis; LT: Left Temporalis. 


\section{DISCUSSION}

In the present study, the anteroposterior skeletal alterations were not statistically significant for either the maxilla or the mandible. In fact, the literature has demonstrated that the use of quadhelix appliances for correction of posterior crossbite does not cause major alterations on the anteroposterior plane $[11,14,16,17]$. No vertical alteration was greater than 1 degree or $1 \mathrm{~mm}$, demonstrating that the vertical alterations after maxillary expansion with the quadhelix appliance do not have clinical significance either. These results are consistent with those of previous studies [11,16,17].

Some reports have indicated a significant maxillary height increase as one of the effects of maxillary expansion with the quadhelix appliance [18]. According to Kecik et al. [19], the vertical skull dimensions of patients treated by slow maxillary expansion with the quadhelix appliance showed a significant increase in maxillary height related to the posterior rotation of the mandible, caused by vertical displacement of the permanent maxillary first molars $[11,19]$. Contrary to these findings, however, the present study did not show expressive alterations in the vertical behavior, which may be explained by the characteristics of the studied population, as all children enrolled in this study were in the mixed dentition phase. Additionally, a short observation period was used.

The alterations in the dental pattern showed a statistically significant increase $(p<0.05)$ of $\underline{1}$-ANS-PNS after maxillary expansion with the quadhelix appliance. This alteration does not seem to be a direct consequence of the maxillary expansion because the values referring to the anteromaxillary height (N-ANS) did not exhibit expressive variations. The extrusion of the maxillary central incisors observed in the present study is consistent with the dentition development phase of the treated patients. The mean age of the patients enrolled in this study (7 years 4 months) is the age at which eruption of the maxillary and mandibular permanent incisors occurs. Therefore, the significant difference of 1-ANS-PNS value is due to the normal development of the occlusion in these patients, rather than to maxillary expansion.

In this study, EMG analysis was used to evaluate the muscular activity pattern produced by the dental alterations resulting from slow maxillary expansion. EMG is a resource that permits the study of the patient as a whole, considering not only the search for occlusal balance by orthodontic mechanics, but also the balance of the stomatognathic system in the analyzed clinical situations. Although several studies have investigated the orthodontic effects of the maxillary expansion [11,13,16-20], the functional effect of this therapy have not been correlated, which substantially limits a direct comparison of the present results.

The mandibular rest position is maintained by the viscoelastic properties of the muscles and tendons, which oppose the gravity force [21]. In the clinical rest condition, EMG activity must be minimal or absent, with spontaneously relaxed muscles $[22,23]$. At rest, the low tonus activity levels of the masticatory muscles are controlled by sensorial receptors and by the central nervous system. In this way, any alteration in the balance of the stomatognathic system would produce changes in muscle tension, as observed at muscular rest [24].

In this study EMG activity was observed for all muscles analyzed in the clinical rest condition at $T_{1}$. This can be explained by the bone and muscle alterations occurring in the stomatognathic system of patients with malocclusion [25]. In patients with stomatognathic system disorders, the mean EMG activity of the masticatory muscles at rest is greater than that of healthy individuals, indicating an increase of the basal tonus [26,27].

In the analysis of EMG activity of patients after the slow maxillary expansion, it was expected that muscle activity would decrease or cease completely in the mandibular rest position, resulting in an adequate maxillomandibular position according to the normal occlusion principles. Although non-significant, it was possible to notice a decrease in EMG activity of the masseter muscles. However, the increase in activity of the temporalis muscles seems to reflect an adaptation and reprogramming of the musculature to the new occlusal condition. Indeed, the temporalis muscles are the main regulators of the mandibular posture in the clinical rest condition $[2,24,25]$. Such increase may also be related to a possible tonus action produced by the quadhelix appliance, which would allow the temporalis muscles to optimize their electric activity, corroborating the results of a previous study [5].

Chewing is one of the most complex, sophisticated and critical neurophysiologic motor mechanisms in humans. It is the initial step in the digestive process and is considered one of the most important functions of the stomatognathic system [22]. Occlusion is one of the most important factors influencing masticatory function. An adequate occlusion allows the bilateral, alternate and multidirectional chewing. When this occurs, the food is distributed homogeneously on tooth surfaces, both on the right and left sides, with a uniform incidence of masticatory forces in the tooth supporting tissues, which maintains the stability of the periodontal tissues and the occlusion itself.

The children with a posterior crossbite have irregular, complex, reverse or contralateral masticatory cycles, very similar to the masticatory cycle pattern of patients with temporomandibular disorders. After treatment, there is a predominance of more regular and symmetric cycles 
similar to those of patients with normal occlusion. The largest number of possible dental contacts is obtained in the maximal habitual intercuspal position, resulting in a greater masticatory force due to the increase of the contact area in occlusion. The number of dental contacts strongly influences the EMG activity, as young individuals with fewer dental contacts have been shown to present lower EMG activity in the masticatory muscles $[2,25]$.

After correction of posterior crossbite, the EMG activity of all muscles evaluated in the present study decreased considerably during habitual chewing. The smaller number of dental contacts established at $\mathrm{T}_{2}$ could produce a decrease of EMG activity of the masticatory muscles due to the need to perform an overcorrection of posterior crossbite, as reported elsewhere [2,25]. Occlusal instability and modifications in the dentition are determinant factors in muscular alterations noticed after the orthodontic treatment.

Muscular alterations that occur during and after orthodontic therapy are important factors in the morphological and aesthetic changes, and in treatment stabilization. The lack of muscular adaptation to occlusal changes may lead to recurrences of the treatment because the anatomic and functional structures of the stomatognathic system are interdependent and tend to seek a balanced condition. There is therefore the need for an appropriate retention period after correction of a malocclusion in such a way that the musculature can adjust to the new tooth positions. Future studies are required to assess whether the functional changes observed in this investigation resulted from either treatment or craniofacial growth, and to elucidate what would occur with the musculature in the follow up period after completion of the orthodontic treatment.

Maxillary expansion performed with the quadhelix appliance in children did not produce significant vertical and anteroposterior cephalometric skeletal alterations. Among the cephalometric variables related to the dental behavior, only the 1 -ANS-PNS values presented a significant increase at the end of treatment due to normal development of the occlusion.

At muscular rest, the EMG analysis exhibited a significant increase in temporalis muscle activity after removal of the quadhelix appliance, which suggests an adaptation and reprogramming of the musculature to the new occlusal condition. During habitual chewing, all evaluated muscles presented a significant decrease in EMG activity, probably due to the smaller number of dental contacts established in the posttreatment phase.

\section{REFERENCES}

[1] O’Bryan, B.L., Sadowsky, C., Schneider, B. and Begole,
E. (1995) An evaluation of mandibular asymmetry in adults with unilateral posterior crossbite. American Journal of Orthodontics \& Dentofacial Orthopedics, 107, 394-400. doi:10.1016/S0889-5406(95)70092-7

[2] Ferrario, V.F., Sforza, C., Serrao, G., Colombo, A. and Schmitz, J.H. (1999) The effects of a single intercuspal interference on electromyographic characteristics of human masticatory muscles during maximal voluntary teeth clenching. Cranio, 17, 184-188.

[3] Bakke, M., Michler, L. and Moller, E. (1992) Occlusal control of mandibular elevator muscles. Scandinavian Journal of Dental Research, 100, 284-291.

[4] Bazzotto, L. (1998) Mandible position and head posture: Electromyography of sternocleidomastoids. Cranio, 16, 100-108.

[5] Troelstrup, B. and Möller, E. (1970) Electromyography of the temporalis and masseter muscles in children with unilateral cross-bite. Scandinavian Journal of Dental Research, 78, 425-430.

[6] Ingervall, B. and Thilander, B. (1975) Activity of temporal and masseter muscles in children with a lateral forced bite. The Angle Orthodontist, 45, 249-258.

[7] Thilander, B., Wahlund, S. and Lennartsson, B. (1984) The effect of early interceptive treatment in children with posterior cross-bite. European Journal of Orthodontics, 6, 25-34. doi:10.1093/ejo/6.1.25

[8] Silva Filho, O.G., Valladares Neto, J. and Almeida, R.R. (1989) Early correction of posterior crossbite: Biomechanical characteristics of the appliances. The Journal of Pedodontics, 13, 195-221.

[9] McNamara, J.A. (2002) Early intervention in the transverse dimension: Is it worth the effort? American Journal of Orthodontics \& Dentofacial Orthopedics, 121, 572-574. doi: $10.1067 / \bmod .2002 .124167$

[10] Godoy, F., Godoy-Bezerra, J. and Rosenblatt, A. (2011) Treatment of posterior crossbite comparing 2 appliances: A community-based trial. American Journal of Orthodontics \& Dentofacial Orthopedics, 139, 45-52. doi:10.1016/j.ajodo.2010.06.017

[11] Hicks, E.P. (1978) Slow maxillary expansion: A clinical study of the skeletal versus dental response to low-magnitude force. American Journal of Orthodontics, 73, 121141. doi:10.1016/0002-9416(78)90183-5

[12] Asher, C. (1985) The removable quad-helix appliance. British Journal of Orthodontics, 12, 40-45.

[13] Bjerklin, K. (2000) Follow-up control of patients with unilateral posterior cross-bite treated with expansion plates or the quad-helix appliance. Journal of Orofacial Orthopedics, 61, 112-124. doi:10.1007/BF01300353

[14] Herold, J.S. (1989) Maxillary expansion: A retrospective study of three methods of expansion and their long-term sequelae. British Journal of Orthodontics, 16, 195-200.

[15] Houston, W.J.B. (1983) The analysis of errors in orthodontic measurements. American Journal of Orthodontics, 83, 382-390. doi:10.1016/0002-9416(83)90322-6

[16] Sandikçioglu, M. and Hazar, S. (1997) Skeletal and dental changes after maxillary expansion in the mixed denti- 
tion. American Journal of Orthodontics \& Dentofacial Orthopedics, 111, 321-327.

doi:10.1016/S0889-5406(97)70191-4

[17] Erdinç, A.E., Ugur, T. and Erbay, E. (1999) A comparison of different treatment techniques for posterior crossbite in the mixed dentition. American Journal of Orthodontics \& Dentofacial Orthopedics, 116, 287-300. doi:10.1016/S0889-5406(99)70240-4

[18] Frank, S.W. and Engel, G.A. (1982) The effects of maxillary quad-helix appliance expansion on cephalometric measurements in growing orthodontic patients. American Journal of Orthodontics, 81, 378-389. doi:10.1016/0002-9416(82)90075-6

[19] Kecik, D., Kocadereli, I. and Saatci, I. (2007) Evaluation of the treatment changes of functional posterior crossbite in the mixed dentition. American Journal of Orthodontics \& Dentofacial Orthopedics, 131, 202-215. doi:10.1016/j.ajodo.2005.03.030

[20] Huynh, T., Kennedy, D.B., Joondeph, D.R. and Bollen, A.M. (2009) Treatment response and stability of slow maxillary expansion using Haas, hyrax, and quad-helix appliances: A retrospective study. American Journal of Orthodontics \& Dentofacial Orthopedics, 136, 331-339. doi:10.1016/j.ajodo.2007.08.026

[21] Voudouris, J.C. and Kuftinec, M.M. (2000) Improved clinical use of Twin-block and Herbst as a result of radiating viscoelastic tissue forces on the condyle and fossa in treatment and long-term retention: Growth relativity. American Journal of Orthodontics \& Dentofacial Ortho- pedics, 117, 247-266. doi:10.1016/S0889-5406(00)70231-9

[22] Vitti, M. and Basmajian, J.V. (1975) Muscles of mastication in small children. American Journal of Orthodontics, 68, 412-419. doi:10.1016/0002-9416(75)90182-7

[23] Carr, A.B. (1991) Postural contractile activities of human jaw muscles following use of an occlusal splint. Journal of Oral Rehabilitation, 18, 185-191. doi:10.1111/j.1365-2842.1991.tb00047.x

[24] Harper, R.P., Bruin, H. and Burcea, I. (1997) Muscle activity during mandibular movements in normal and mandibular retrognathic subjects. Journal of Oral and Maxillofacial Surgery, 55, 225-233. doi:10.1016/S0278-2391(97)90530-9

[25] Ferrario, V.F., Sforza, C. and Serrao, G. (1999) The influence of crossbite on the coordinated eletromyographic activity of human masticatory muscles during mastication. Journal of Oral Rehabilitation, 26, 575-581. doi:10.1046/j.1365-2842.1999.00419.x

[26] Liu, Z.J., Yamagata, K., Kasahara, Y. and Ito, G. (1999) Electromyographic examination of jaw muscles in relation to symptoms and occlusion of patients with temporomandibular joint disorders. Journal of Oral Rehabilitation, 26, 33-47. doi:10.1046/j.1365-2842.1999.00356.x

[27] Pinho, J.C., Caldas, F.M., Mora, M.J. and Santana-Penin, U. (2000) Electromyographic activity in patients with temporomandibular disorders. Journal of Oral Rehabilitation, 27, 985-990.

doi:10.1046/j.1365-2842.2000.00571.x 